\title{
Competitive Exploration of Rectilinear Polygons
}

\author{
Mikael Hammar* Bengt J. Nilsson ${ }^{\dagger} \quad$ Mia Persson $^{\dagger}$
}

\begin{abstract}
Exploring a polygon with robots, when the robots do not have knowledge of the surroundings can be viewed as an online problem. Typical for online problems is that decisions must be made based on past events without complete information about the future. In our case the robots do not have complete information about the environment. Competitive analysis can be used to measure the performance of methods solving online problems. The competitive ratio of such a method is the ratio between the method's performance and the performance of the best method having full knowledge of the future. We are interested in obtaining good bounds on the competitive ratio of exploring polygons and prove constant competitive strategies and lower bounds for exploring a simple rectilinear polygon in the $L_{1}$ metric.
\end{abstract}

\section{Introduction}

Exploring an environment is an important and well studied problem in robotics. In many realistic situations the robots do not possess complete knowledge about the environment, e.g., they may not have a map of the surroundings $[1,2,4,6,7,8,9]$.

The search of the robots can be viewed as an online problem since the robots' decisions about the search are based only on the part of the environment that they have seen so far. We use the framework of competitive analysis to measure the performance of an online search strategy $S$. The competitive ratio of $S$ is defined as the maximum of the ratio of the distance traveled by the robot that moves the farthest using $S$ to the optimal distance of the search.

We are interested in obtaining good bounds for the competitive ratio of exploring a rectilinear polygon. The search is modeled by a path or closed tour followed by one or more point sized robots inside the polygon, given a starting point for the search. The only information that the robots have about the surrounding polygon is the part of the polygon that they together have seen so far.

For the case of exploration with one robot, Deng et al. [4] show a deterministic strategy having competitive ratio two for this problem if distance is measured according to the $L_{1}$-metric. Hammar et al. [5] prove a strategy with competitive ratio $5 / 3$ and Kleinberg [7] proves a lower bound of $5 / 4$ for the competitive ratio of any deterministic strategy. We will show a deterministic strategy obtaining a competitive ratio of $3 / 2$ for searching a rectilinear polygon in the $L_{1}$-metric with one robot.

\footnotetext{
${ }^{*}$ Department of Computer Science, Salerno University, Baronissi (SA) - 84081, Italy. email: hammar@dia.unisa.it

${ }^{\dagger}$ Technology and Society, Malmö University College, S-205 06 Malmö, Sweden.

email: \{Bengt.Nilsson, Mia.Persson\}@ts.mah.se
} 
We also present a lower bound of 2 for the corresponding path exploration problem for one robot in rectilinear polygons and competitive results on exploration with two and three robots.

The paper is organized as follows. In the next section we present some definitions and preliminary results. In Section 3 we give an overview of the strategy by Deng et al. [4]. Section 4 contains an improved strategy for single robot exploration giving a competitive ratio of $3 / 2$. In sections 5 and 6 we consider path exploration and exploration with multiple robots.

\section{Preliminaries}

We will henceforth always measure distance according to the $L_{1}$ metric, i.e., the distance between two points $p$ and $q$ is defined by

$$
\|p, q\|=\left|p_{x}-q_{x}\right|+\left|p_{y}-q_{y}\right|,
$$

where $p_{x}$ and $q_{x}$ are the $x$-coordinates of $p$ and $q$ and $p_{y}$ and $q_{y}$ are the $y$-coordinates. We define the $x$-distance between $p$ and $q$ to be $\|p, q\|_{x}=\left|p_{x}-q_{x}\right|$ and the $y$-distance to be $\|p, q\|_{y}=\left|p_{y}-q_{y}\right|$.

If $C$ is a polygonal curve, then the length of $C$, denoted length $(C)$, is defined the sum of the distances between consecutive pairs of segment end points in $C$.

Let $\mathbf{P}$ be a simple rectilinear polygon. Two points in $\mathbf{P}$ are said to see each other, or be visible to each other, if the line segment connecting the points lies in $\mathbf{P}$. Let $p$ be a point somewhere inside $\mathbf{P}$. A watchman route through $p$ is defined to be a closed curve $C$ that passes through $p$ such that every point in $\mathbf{P}$ is seen by some point on $C$. The shortest watchman route through $p$ is denoted by $S W R_{p}$. It can be shown that the shortest watchman route in a simple polygon is a closed polygonal curve [3].

Since we are only interested in the $L_{1}$ length of a polygonal curve we can assume that the curve is rectilinear, that is, the segments of the curve are all axis parallel. Note that the shortest rectilinear watchman route through a point $p$ is not necessarily unique.

For a point $p$ in $\mathbf{P}$ we define four quadrants with respect to $p$. Those are the regions obtained by cutting $\mathbf{P}$ along the two maximal axis parallel line segments that pass through $p$. The four quadrants are denoted $\mathbf{Q}_{\mathbf{1}}(p), \mathbf{Q}_{2}(p), \mathbf{Q}_{3}(p)$, and $\mathbf{Q}_{4}(p)$ in anti-clockwise order from the top right quadrant to the bottom right quadrant. We let $\mathbf{Q}_{i, j}(p)$ denote the union of $\mathbf{Q}_{i}(p)$ and $\mathbf{Q}_{j}(p)$.

Consider a reflex vertex of $\mathbf{P}$. The two edges of $\mathbf{P}$ connecting at the reflex vertex can each be extended inside $\mathbf{P}$ until the extensions reach a boundary point. The segments thus constructed are called extensions and to each extension a direction is associated. The direction is the same as that of the collinear polygon edge as we follow the boundary of $\mathbf{P}$ in clockwise order. We use the four compass directions north, west, south, and east to denote the direction of an extension.

Lemma 2.1 (Chin, Ntafos [3]) A closed curve is a watchman route for $\mathbf{P}$ if and only if the curve has at least one point to the right of every extension of $\mathbf{P}$.

Our first objective is to present a competitive online strategy that enables a robot to follow a closed curve from the start point $s$ in $\mathbf{P}$ and back to $s$ with the curve being a watchman route for $\mathbf{P}$.

An extension $e$ splits $\mathbf{P}$ into two sets $\mathbf{P}_{l}$ and $\mathbf{P}_{r}$ with $\mathbf{P}_{l}$ to the left of $e$ and $\mathbf{P}_{r}$ to the right. We say a point $p$ is to the left of $e$ if $p$ belongs to $\mathbf{P}_{l}$. To the right is defined analogously.

As a further definition we say that an extension $e$ is a left extension with respect to a point $p$, if $p$ lies to the left of $e$, and an extension $e$ dominates another extension $e^{\prime}$, if all points of $\mathbf{P}$ to the right 


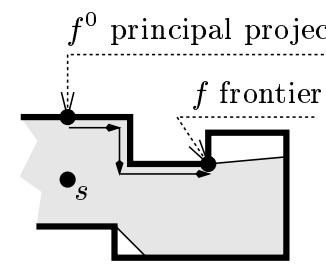

(a)

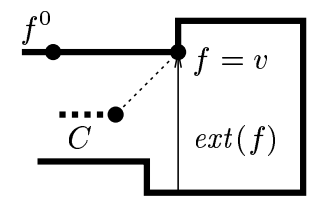

(b)

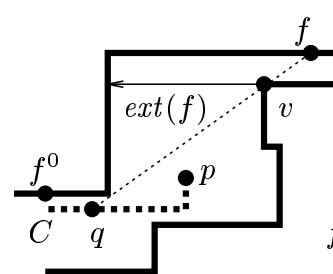

(c)

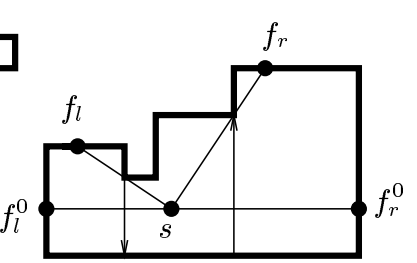

(d)

Figure 1: Illustrating definitions.

of $e$ are also to the right of $e^{\prime}$. By Lemma 2.1 we are only interested in the extensions that are left extensions with respect to the starting point $s$ since the other ones already have a point (the point $s$ ) to the right of them. So without loss of clarity when we mention extensions we will always mean extensions that are left extensions with respect to $s$.

\section{An Overview of GO}

Consider a rectilinear polygon $\mathbf{P}$ that is not a priori known to the robot. Let $s$ be the robot's initial position inside $\mathbf{P}$. For the starting position $s$ of the robot we associate a point $f^{0}$ on the boundary of $\mathbf{P}$ that is visible from $s$ and call $f^{0}$ the principal projection point of $s$. For instance, we can choose $f^{0}$ to be the first point on the boundary that is hit by an upward ray starting at $s$. Let $f$ be the end point of the boundary that the robot sees as we scan the boundary of $\mathbf{P}$ in clockwise order; see Figure 1(a). The point $f$ is called the current frontier.

Let $C$ be a polygonal curve starting at $s$. Formally a frontier $f$ of $C$ is a vertex of the visibility polygon, $\mathbf{V P}(C)$ of $C$ adjacent to an edge $e$ of $\mathbf{V P}(C)$ that is not an edge of $\mathbf{P}$. Extend $e$ until it hits a point $q$ on $C$ and let $v$ be the vertex of $\mathbf{P}$ that is first encountered as we move along the line segment $[q, f]$ from $q$ to $f$. We denote the left extension with respect to $s$ associated to the vertex $v$ by $\operatorname{ext}(f)$; see Figures 1(b) and (c).

Deng et al. [4] introduce an online strategy called greedy-online, GO for short, to explore a simple rectilinear polygon $\mathbf{P}$ in the $L_{1}$ metric. If the starting point $s$ lies on the boundary of $\mathbf{P}$, their strategy, we call it BGO, goes as follows: from the starting point scan the boundary clockwise and establish the first frontier $f$. Move to the closest point on $\operatorname{ext}(f)$ and establish the next frontier. Continue in this fashion until all of $\mathbf{P}$ has been seen and move back to the starting point.

Deng et al. show that a robot using strategy BGO to explore a rectilinear polygon follows a tour with shortest length, i.e., BGO has competitive ratio one. They also present a similar strategy, called IGO, for the case when the starting point $s$ lies in the interior of $\mathbf{P}$. For IGO they show a competitive ratio of two, i.e., IGO specifies a tour that is at most twice as long as the shortest watchman route through $s$.

IGO shoots a ray upwards to establish a principal projection point $f^{0}$ and then scans the boundary clockwise to obtain the frontier. Next, it proceeds exactly as BGO, moving to the closest point on the extension of the frontier, updating the frontier, and repeating the process until all of the polygon has been seen. 
It is clear that BGO could just as well scan the boundary anti-clockwise instead of clockwise when establishing the frontiers and still have the same competitive ratio. Hence, BGO can be seen as two strategies, one scanning clockwise and the other anti-clockwise. We can therefore parameterize the two strategies so that $\mathrm{BGO}(p$, orient $)$ is the strategy beginning at some point $p$ on the boundary and scanning with orientation orient where orient is either clockwise $c w$ or anti-clockwise $a w$.

Similarly for IGO, we can not only choose to scan clockwise or anti-clockwise for the frontier but also choose to shoot the ray giving the first principal projection point in any of the four compass directions north, west, south, or east. Thus IGO in fact becomes eight different strategies that we can parameterize as $\operatorname{IGO}(p$, dir, orient $)$ and the parameter dir can be one of north, south, west, or east.

We further define partial versions of GO starting at boundary and interior points. Strategies $\operatorname{PBGO}(p$, orient, region $)$ and $\mathrm{PIGO}(p$, dir, orient, region $)$ apply GO until either the robot has explored all of region or the robot leaves the region region. The strategies return as result the position of the robot when it leaves region or when region has been explored. Note that $\operatorname{PBGO}(p$, orient, $\mathbf{P})$ and $\operatorname{PIGO}(p$, dir, orient, $\mathbf{P})$ are the same strategies as $\mathrm{BGO}(p$, orient $)$ and $\operatorname{IGO}(p$, dir, orient $)$ respectively except that they do not move back to $p$ when all of $\mathbf{P}$ has been seen.

\section{The Strategy CGO}

We present a new strategy competitive-greedy-online (CGO) that explores two quadrants simultaneosly without using up too much distance. We assume that $s$ lies in the interior of $\mathbf{P}$ since otherwise we can use BGO and achieve an optimal route. The strategy uses two frontier points simultaneously to improve the competitive ratio. However, to initiate the exploration, the strategy begins by performing a scan of the polygon boundary to decide in which direction to start the exploration. This is to minimize the loss inflicted upon us by our choice of initial direction.

The initial scan works as follows: construct the visibility polygon $\mathbf{V P}(s)$ of the initial point $s$. Consider the set of edges in $\mathbf{V P}(s)$ not coinciding with the boundary of $\mathbf{P}$. The end points of these edges define a set of frontier points each having an associated left extension. Let $e$ denote the left extension that is furthest from $s$ (distance being measured orthogonally to the extension). Let $l$ be the infinite line through $e$. We rotate the view point of $s$ so that $\mathbf{Q}_{3}(s)$ and $\mathbf{Q}_{4}(s)$ intersect $l$ whereas $\mathbf{Q}_{1}(s)$ and $\mathbf{Q}_{2}(s)$ do not. Hence, $e$ is a horizontal extension lying below $s$. The initial direction of exploration is upwards through $\mathbf{Q}_{1}(s)$ and $\mathbf{Q}_{2}(s)$. The two frontier points used by the strategy are obtained as follows: the left frontier $f_{l}$ is established by shooting a ray towards the left for the left principal projection point $f_{l}^{0}$ and then scan the boundary in clockwise direction for $f_{l}$; see Figure $1(\mathrm{~d})$. The right frontier $f_{r}$ is established by shooting a ray towards the right for the right principal projection point $f_{r}^{0}$ and then scan the boundary in anti-clockwise direction for $f_{r}$; see Figure 1(d). To each frontier point we associate a left extension $\operatorname{ext}\left(f_{l}\right)$ and a right extension $\operatorname{ext}\left(f_{r}\right)$ with respect to $s$.

The strategy CGO, presented in pseudo code below makes use of three different substrategies: CGO-0, CGO-1, and CGO-2, that each takes care of specific cases that can occur. Subsequently we will prove the correctness and competitive ratio for each of the substrategies.

Our strategy ensures that whenever it performs one of the substrategies this is the last time that the outermost while-loop is executed. Hence, the loop is repeated only when the strategy does not enter any of the specified substrategies. The loop will lead the strategy to follow a straight line and we will maintain the invariant during the while-loop that all of the region $\mathbf{Q}_{3,4}(p) \cap \mathbf{Q}_{1,2}(s)$ has been 


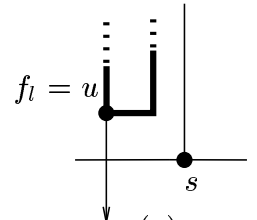

(a)

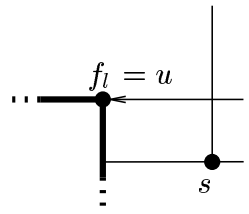

(b)

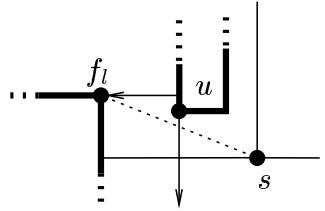

(c)

Figure 2: Illustrating the key point $u$.

seen.

We distinguish four classes of extensions. $\mathcal{A}$ is the class of extensions $e$ whose defining edge is above $e, \mathcal{B}$ is the class of extensions $e$ whose defining edge is below $e$. Similarly, $\mathcal{L}$ is the class of extensions $e$ whose defining edge is to the left of $e$, and $\mathcal{R}$ is the class of extensions $e$ whose defining edge is to the right of $e$. For conciseness, we use $\mathcal{C}_{1} \mathcal{C}_{2}$ as a shorthand for the Cartesian product $\mathcal{C}_{1} \times \mathcal{C}_{2}$ of the two classes $\mathcal{C}_{1}$ and $\mathcal{C}_{2}$.

We define two key vertices $u$ and $v$ together with their extensions $\operatorname{ext}(u)$ and $\operatorname{ext}(v)$ that are useful to establish the correct substrategy to enter. The vertex $u$ lies in $\mathbf{Q}_{2}(s)$ and $v$ in $\mathbf{Q}_{1}(s)$. If $\operatorname{ext}\left(f_{l}\right) \in \mathcal{A} \cup \mathcal{B}$, then $u$ is the vertex issuing $\operatorname{ext}\left(f_{l}\right)$ and $\operatorname{ext}(u)=\operatorname{ext}\left(f_{l}\right)$. If $\operatorname{ext}\left(f_{l}\right) \in \mathcal{L}$ and $\operatorname{ext}\left(f_{l}\right)$ crosses the vertical line through $s$, then $u$ is the vertex issuing $\operatorname{ext}\left(f_{l}\right)$ and again $\operatorname{ext}(u)=\operatorname{ext}\left(f_{l}\right)$. If $\operatorname{ext}\left(f_{l}\right) \in \mathcal{L}$ does not cross the vertical line through $s$, then $u$ is the leftmost vertex of the bottommost edge visible from the robot, on the boundary going from $f_{l}$ clockwise until we leave $\mathbf{Q}_{2}(s)$. The extension $\operatorname{ext}(u)$ is the left extension issued by $u$, and hence, $\operatorname{ext}(u) \in \mathcal{A}$; see Figures 2(a), (b), and (c). The vertex $v$ is defined symmetrically in $\mathbf{Q}_{1}(s)$ with respect to $f_{r}$.

Each of the substrategies is presented in sequence and for each of them we prove that if CGO executes the substrategy, then the competitive ratio of CGO is bounded by $3 / 2$. Let $F R_{s}$ be the closed route followed by strategy CGO starting at an interior point $s$. Let $F R_{s}(p, q$, orient) denote the subpath of $F R_{s}$ followed in direction orient from point $p$ to point $q$, where orient can either be $c w$ (clockwise) or $a w$ (anti-clockwise). Similarly, we define the subpath $S W R_{s}\left(p, q\right.$, orient) of $S W R_{s}$. We denote by $S P(p, q)$ a shortest rectilinear path from $p$ to $q$ inside $\mathbf{P}$.

We begin by establishing two simple but useful lemmas.

LEMMA 4.1 If $t$ is a point on some tour $S W R_{s}$, then length $\left(S W R_{t}\right) \leq$ length $\left(S W R_{s}\right)$.

Proof: Since $S W R_{s}$ passes through $t$, the route is a watchman route through $t$. But since $S W R_{t}$ is the shortest watchman route through $t$, the lemma follows.

LEMMA 4.2 Let $S$ be a set of points that are enclosed by some tour $S W R_{s}$, and let $S_{1}=S \cap \mathbf{Q}_{1,2}(s)$, $S_{2}=S \cap \mathbf{Q}_{2,3}(s), S_{3}=S \cap \mathbf{Q}_{3,4}(s)$, and $S_{4}=S \cap \mathbf{Q}_{1,4}(s)$. Then

$$
\text { length }\left(S W R_{s}\right) \geq 2 \max _{p \in S_{1}}\left\{\|s, p\| \|_{y}\right\}+2 \max _{p \in S_{2}}\left\{\|s, p\|_{x}\right\}+2 \max _{p \in S_{3}}\left\{\|s, p\|_{y}\right\}+2 \max _{p \in S_{4}}\left\{\|s, p\|_{x}\right\} .
$$

Proof: $S W R_{s}$ encloses all the points in $S$ and since we calculate length according to the $L_{1}$ metric, the smallest tour enclosing the points is the smallest rectangle containing them. The length of the rectangle's perimeter is as stated, proving the result. 
The structure of the following proofs are very similar to each other. In each case we will establish a point $t$ that we can ensure is passed by $S W R_{s}$ and that either lies on the boundary of $\mathbf{P}$ or can be viewed as to lie on the boundary of $\mathbf{P}$. We then consider the tour $S W R_{t}$ and compare its length the length of $F R_{s}$. By Lemma 4.1 we know that length $\left(S W R_{t}\right) \leq \operatorname{length}\left(S W R_{s}\right)$, hence the difference in length between $F R_{s}$ and $S W R_{t}$ is an upper bound on the loss produced by CGO.

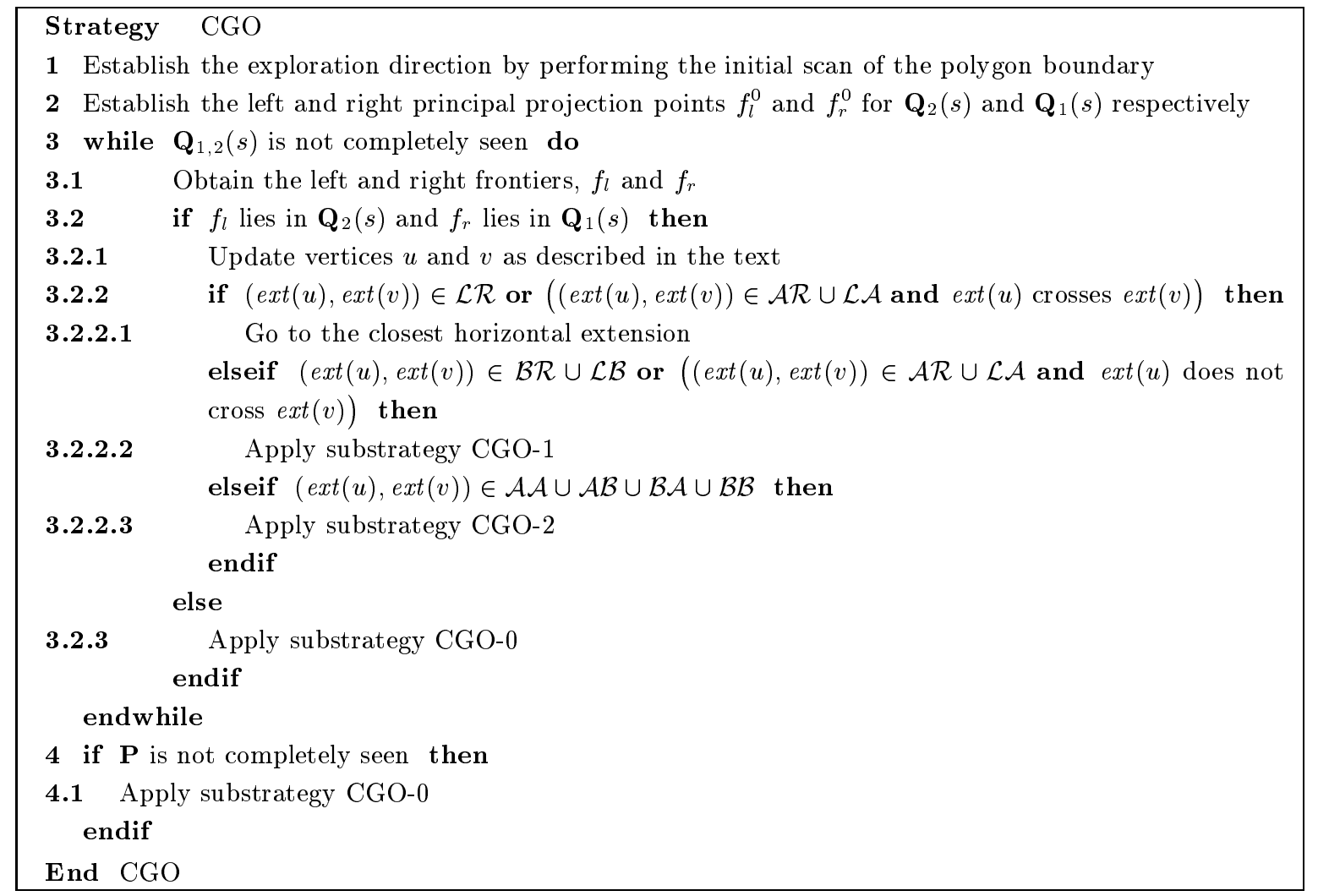

We start by presenting CGO-0, that does the following: Let $p$ be the current robot position. If $\mathbf{Q}_{1}(p)$ is completely seen from $p$ then we run $\operatorname{PIGO}(p$, north $, a w, \mathbf{P})$ and move back to the starting point $s$, otherwise $\mathbf{Q}_{2}(p)$ is completely seen from $p$ and we run $\operatorname{PIGO}(p$, north, $c w, \mathbf{P})$ and move back to the starting point $s$.

Lemma 4.3 If the strategy applies substrategy CGO-0, then length $\left(F R_{s}\right)=\operatorname{length}\left(S W R_{s}\right)$.

Proof: Assume that CGO-0 realizes that when $F R_{s}$ reaches the point $p$, then $\mathbf{Q}_{1}(p)$ is completely seen from $p$. The other case, that $\mathbf{Q}_{2}(p)$ is completely seen from $p$ is symmetric.

Since the path $F R_{s}(s, p$, orient $)$ that the strategy has followed when it reaches point $p$ is a straight line, the point $p$ is the currently topmost point of the path. Hence, we can add a vertical spike issued by the boundary point immediately above $p$, giving a new polygon $\mathbf{P}^{\prime}$ having $p$ on the boundary and furthermore with the same shortest watchman route through $p$ as $\mathbf{P}$. This means that performing strategy $\operatorname{IGO}(p$, north, orient $)$ in $\mathbf{P}$ yields the same result as performing $\mathrm{BGO}(p$, orient $)$ in $\mathbf{P}^{\prime}, p$ being 
a boundary point in $\mathbf{P}^{\prime}$, and orient being either $c w$ or $a w$. The tour followed is therefore a shortest watchman route through the point $p$ in both $\mathbf{P}^{\prime}$ and $\mathbf{P}$.

Also the point $p$ lies on an extension with respect to $s$, by the way $p$ is defined, and it is the closest point to $s$ such that all of $\mathbf{Q}_{1}(s)$ has been seen by the path $F R_{s}(s, p$, orient $)=S P(s, p)$. Hence, there is a route $S W R_{s}$ that contains $p$ and by Lemma $4.1 \operatorname{length}\left(S W R_{p}\right) \leq \operatorname{length}\left(S W R_{s}\right)$. The tour followed equals $F R_{s}=S P(s, p) \cup S W R_{p}(p, s, a w)$, and we have that $\operatorname{length}\left(F R_{s}\right)=\operatorname{length}\left(S W R_{p}\right) \leq$ length $\left(S W R_{s}\right)$, and since $F R_{s}$ cannot be strictly shorter than $S W R_{s}$ the equality holds which concludes the proof.

Next we present CGO-1. Let $u$ and $v$ be the key vertices as defined earlier. The strategy does the following: if $(\operatorname{ext}(u), \operatorname{ext}(v)) \in \mathcal{L A} \cup \mathcal{L B}$, we mirror the polygon $\mathbf{P}$ at the vertical line through $s$ and swap the names of $u$ and $v$. Hence, $(\operatorname{ext}(u), \operatorname{ext}(v)) \in \mathcal{A R} \cup \mathcal{B R}$. We continue moving upwards updating $f_{r}$ and $v$ until either all of $\mathbf{Q}_{1}(s)$ has been seen or $\operatorname{ext}(v)$ no longer crosses the vertical line through $s$.

If all of $\mathbf{Q}_{1}(s)$ has been seen then we explore the remaining part of $\mathbf{P}$ using $\operatorname{PIGO}(p$, east, aw, $\mathbf{P})$, where $p$ is the current robot position.

If $\operatorname{ext}(v)$ no longer crosses the vertical line through $s$ then we either need to continue the exploration by moving to the right or return to $u$ and explore the remaining part of the polygon from there.

If $\|s, p\|_{y}+\|s, u\|_{x} \leq\|s, v\|_{x}$ we choose to return to $u$. If $\operatorname{ext}(u) \in \mathcal{A}$ we run $\operatorname{PBGO}(u, a w, \mathbf{P})$ and if $\operatorname{ext}(u) \in \mathcal{B}$ we use $\operatorname{PBGO}(u, c w, \mathbf{P})$; see Figure 3. Otherwise, $\|s, p\|_{y}+\|s, u\|_{x}>\|s, v\|_{x}$ and in this case we move to the closest point $v^{\prime}$ on $\operatorname{ext}(v)$. By definition, the extension of $v$ is either in $\mathcal{A}$ or $\mathcal{B}$ in this case.

If $\operatorname{ext}(v) \in \mathcal{B}$ then $v=v^{\prime}$ and we choose to run $\operatorname{PBGO}(v, a w, \mathbf{P})$. Otherwise, ext $(v) \in \mathcal{A}$. If $\mathbf{Q}_{1}\left(v^{\prime}\right)$ is seen from $v^{\prime}$ then the entire quadrant has been explored and we run $\operatorname{PIGO}\left(v^{\prime}\right.$, east, aw, $\left.\mathbf{P}\right)$ to explore the remainder of the polygon. If $\mathbf{Q}_{1}\left(v^{\prime}\right)$ is not seen from $v^{\prime}$ then there are still things hidden from the robot in $\mathbf{Q}_{\mathbf{1}}(v)$. We explore the rest of the quadrant using $\operatorname{PBGO}\left(v^{\prime}\right.$, north, aw, $\left.\mathbf{Q}_{1}(v)\right)$ reaching a point $q$ where a second decision needs to be made.

If $v$ is seen from the starting point and $\|s, q\|_{x} \leq\|s, v\|$, we go back to $v$ and run $\operatorname{PBGO}(v, a w, \mathbf{P})$, otherwise we run $\operatorname{PIGO}(q$, east, $c w, \mathbf{P})$ from the interior point $q$; see Figure 5 .

If $v$ is not seen from the starting point $s$ then we go back to $v$ and $\operatorname{run} \operatorname{PBGO}(v, a w, \mathbf{P})$.

To finish the substrategy CGO-1 our last step is to return to the starting point $s$.

Lemma 4.4 If the strategy applies substrategy CGO-1, then length $\left(F R_{s}\right) \leq \frac{3}{2} \operatorname{length}\left(S W R_{s}\right)$.

Proof: We handle each case separately. Assume for the first case that when $F R_{s}$ reaches the point $p$, then $\mathbf{Q}_{1}(p)$ is completely visible. Hence, we have the same situation as in the proof of Lemma 4.3 and using the same proof technique it follows that length $\left(F R_{s}\right)=\operatorname{length}\left(S W R_{s}\right)$.

Assume for the second case that CGO-1 decides to go back to $u$, i.e., that $\|s, p\|_{y}+\|s, u\|_{x} \leq\|s, v\|_{x}$; see Figures 3(a) and (b). The tour followed equals one of

$$
F R_{s}=\left\{\begin{array}{l}
S P(s, p) \cup S P(p, u) \cup S W R_{u} \cup S P(u, s) \\
S P(s, p) \cup S P(p, u) \cup S W R_{u}(u, r, c w) \cup S P(r, s)
\end{array}\right.
$$

where $r$ is the last intersection point of $F R_{s}$ with the horizontal line through $s$. Using that $\|s, p\|_{y}+$ $\|s, u\|_{x} \leq\|s, v\|_{x}$ it follows that the length of $F R_{s}$ in both cases is bounded by

$$
\operatorname{length}\left(F R_{s}\right)=\|s, p\|+\|p, u\|+\operatorname{length}\left(S W R_{u}\right)+\|u, s\|=\operatorname{length}\left(S W R_{u}\right)+2\|s, p\|_{y}+2\|s, u\|_{x}
$$




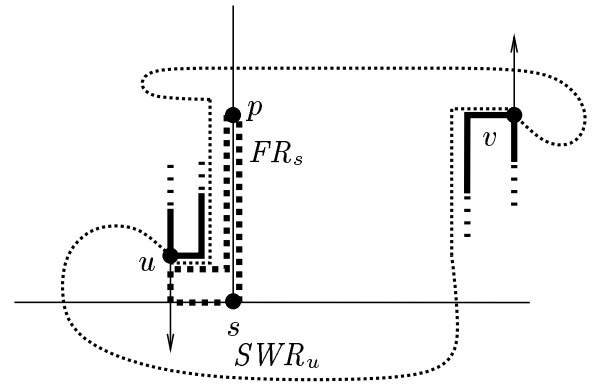

(a)

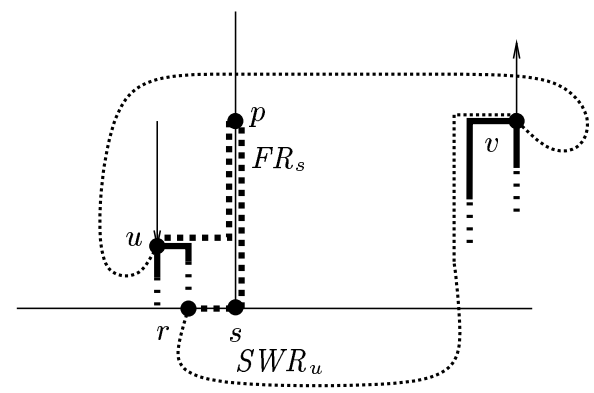

(b)

Figure 3: Illustrating the cases in Lemma 4.4 when $\|s, p\|_{y}+\|s, u\|_{x} \leq\|s, v\|_{x}$.

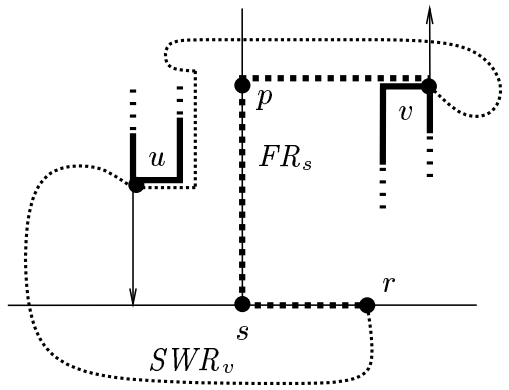

(a)

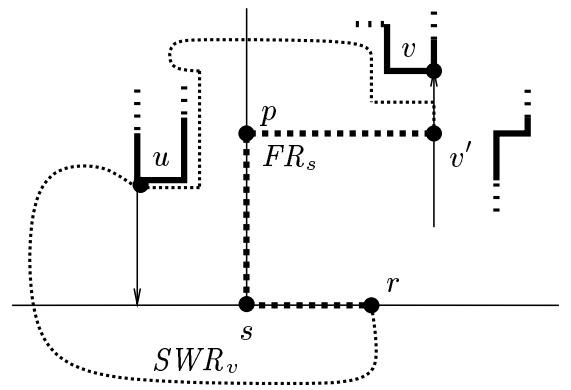

(b)

Figure 4: Illustrating the proof of Lemma 4.4 when $\|s, p\|_{y}+\|s, u\|_{x}>\|s, v\|_{x}$.

$$
\leq \operatorname{length}\left(S W R_{s}\right)+\|s, p\|_{y}+\|s, u\|_{x}+\|s, v\|_{x} \leq \frac{3}{2} \operatorname{length}\left(S W R_{s}\right) .
$$

The inequalities follow from the assumption together with Lemmas 4.1 and 4.2.

Assume for the third case that CGO-1 goes to the right, i.e., that $\|s, p\|_{y}+\|s, u\|_{x}>\|s, v\|_{x}$. We begin by handling the different subcases that are independent of whether $s$ sees $v$; see Figures 4 (a) and (b). The tour followed equals one of

$$
F R_{s}=\left\{\begin{array}{l}
S P(s, v) \cup S W R_{v}(v, r, a w) \cup S P(r, s) \\
S P\left(s, v^{\prime}\right) \cup S W R_{v^{\prime}}\left(v^{\prime}, r, a w\right) \cup S P(r, s)
\end{array}\right.
$$

Since $\|s, v\|_{x}=\left\|s, v^{\prime}\right\|_{x}$ the length of $F R_{s}$ is in both subcases bounded by

$$
\begin{aligned}
\text { length }\left(F R_{s}\right) & \leq \text { length }\left(S W R_{s}\right)+2\|s, v\|_{x}<\operatorname{length}\left(S W R_{s}\right)+\|s, p\|_{y}+\|s, u\|_{x}+\|s, v\|_{x} \\
& \leq \frac{3}{2} \operatorname{length}\left(S W R_{s}\right)
\end{aligned}
$$

The inequalities follow from Lemmas 4.1 and 4.2. 
Assume now that CGO-1 goes to the right, i.e., that $\|s, p\|_{y}+\|s, u\|_{x}>\|s, v\|_{x}$ and that $v$ is indeed seen from $s$; see Figures $5(\mathrm{a})$ and (b). The tour followed in this case is one of

$$
F R_{s}=\left\{\begin{array}{l}
S P(s, v) \cup S W R_{v}(v, q, c w) \cup S P(q, v) \cup S W R_{v}(v, r, a w) \cup S P(r, s) \\
S P(s, v) \cup S W R_{v} \cup S P(v, s)
\end{array}\right.
$$

where $q$ is the resulting location after exploring $\mathbf{Q}_{1}(v)$. Here we use that $v$ is seen from $s$, and hence, that the initial scan guarantees that there is a point $t$ of $S W R_{s}$ in $\mathbf{Q}_{3,4}(s)$ such that $\|s, t\|_{y} \geq\|s, v\|_{x}$, thus $F R_{s}$ is bounded by

$$
\begin{aligned}
\operatorname{length}\left(F R_{s}\right) & =\operatorname{length}\left(S W R_{v}\right)+2 \min \left\{\|s, v\|,\|s, q\|_{x}\right\} \\
& \leq \operatorname{length}\left(S W R_{s}\right)+\|s, v\|_{y}+\|s, v\|_{x}+\|s, q\|_{x} \\
& <\operatorname{length}\left(S W R_{s}\right)+\|s, v\|_{y}+\|s, t\|_{y}+\|s, q\|_{x}+\|s, u\|_{x} \leq \frac{3}{2} \operatorname{length}\left(S W R_{s}\right) .
\end{aligned}
$$

On the other hand, when $v$ is not seen from $s$, the tour follows the path marked with $(*)$ above; see Figure 5(c). Thus, the polygon boundary obscures the view from $s$ to $v$, and hence, there is a point $q^{\prime}$ on the boundary such that the shortest path from $s$ to $v^{\prime}$ contains $q^{\prime}$. The path our strategy follows between $s$ and $v^{\prime}$ is a shortest path and we can therefore assume that it also passed through $q^{\prime}$. We use that $\left\|s, q^{\prime}\right\|_{x} \leq\|s, v\|_{x} \leq\|s, q\|_{x}$ to get the bound.

$$
\begin{aligned}
\text { length }\left(F R_{s}\right) & =\operatorname{length}\left(S W R_{q^{\prime}}\right)+2\left\|s, q^{\prime}\right\|_{x} \leq \operatorname{length}\left(S W R_{s}\right)+\|s, v\|_{x}+\|s, q\|_{x} \\
& <\operatorname{length}\left(S W R_{s}\right)+\|s, v\|_{y}+\|s, u\|_{x}+\|s, q\|_{x} \leq \frac{3}{2} \operatorname{length}\left(S W R_{s}\right) .
\end{aligned}
$$

The inequalities above follow from Lemmas 4.1 and 4.2 and this concludes the proof.

We continue the analysis by first showing the substrategy CGO-2 and then proving its competitive ratio. The strategy does the following: if $\|s, u\|_{x} \leq\|s, v\|_{x}$ then we mirror $\mathbf{P}$ at the vertical line through $s$ also swapping the names of $u$ and $v$. This means that $v$ is closer to the current point $p$ with respect to $x$-distance than $u$. Next, go to $v^{\prime}$, the closest point on $\operatorname{ext}(v)$. If $\operatorname{ext}(v) \in \mathcal{B}, \operatorname{run} \operatorname{PBGO}(v, a w, \mathbf{P})$ since $v=v^{\prime}$. If $\operatorname{ext}(v) \in \mathcal{A}$ and $\mathbf{Q}_{1}(v)$ is seen from $v^{\prime}$ then we run $\operatorname{PIGO}\left(v^{\prime}\right.$, east, aw, $\left.\mathbf{P}\right)$. If $\operatorname{ext}(v) \in \mathcal{A}$ but $\mathbf{Q}_{1}(v)$ is not completely seen from $v^{\prime}$ then we explore $\mathbf{Q}_{1}(v)$ using $\operatorname{PBGO}\left(v^{\prime}\right.$, north $\left., c w, \mathbf{Q}_{1}\left(v^{\prime}\right)\right)$. Once $\mathbf{Q}_{1}(v)$ is explored we have reached a point $q$ and we make a second decision. If $\|s, q\|_{x} \leq\|s, v\|$, go back to $v$ and run $\operatorname{PBGO}(v, a w, \mathbf{P})$, otherwise run $\operatorname{PIGO}(q, e a s t, c w, \mathbf{P})$. Finally go back to $s$.

LEMMA 4.5 If the strategy applies substrategy CGO-2, then length $\left(F R_{s}\right) \leq \frac{3}{2}$ length $\left(S W R_{s}\right)$.

Proof: Assume without loss of generality that $\|s, u\|_{x}>\|s, v\|_{x}$. The other case is proved symmetrically.

Next, assume that $F R_{s}$ passes through $v$; see Figures 6(a), (b), and (c). The tour followed equals one of

$$
F R_{s}=\left\{\begin{array}{l}
S P(s, v) \cup S W R_{v}(v, r, a w) \cup S P(r, s) \\
S P(s, v) \cup S W R_{v}(v, q, c w) \cup S P(q, v) \cup S W R_{v}(v, r, a w) \cup S P(r, s) \\
S P(s, v) \cup S W R_{v} \cup S P(v, s)
\end{array}\right.
$$

where $r$ is the last intersection point of $F R_{s}$ with the horizontal line through $s$. The length of $F R_{s}$ is in each case bounded by

$$
\text { length }\left(F R_{s}\right)=\operatorname{length}\left(S W R_{v}\right)+2 \min \left\{\|s, q\|_{x},\|s, v\|\right\} .
$$



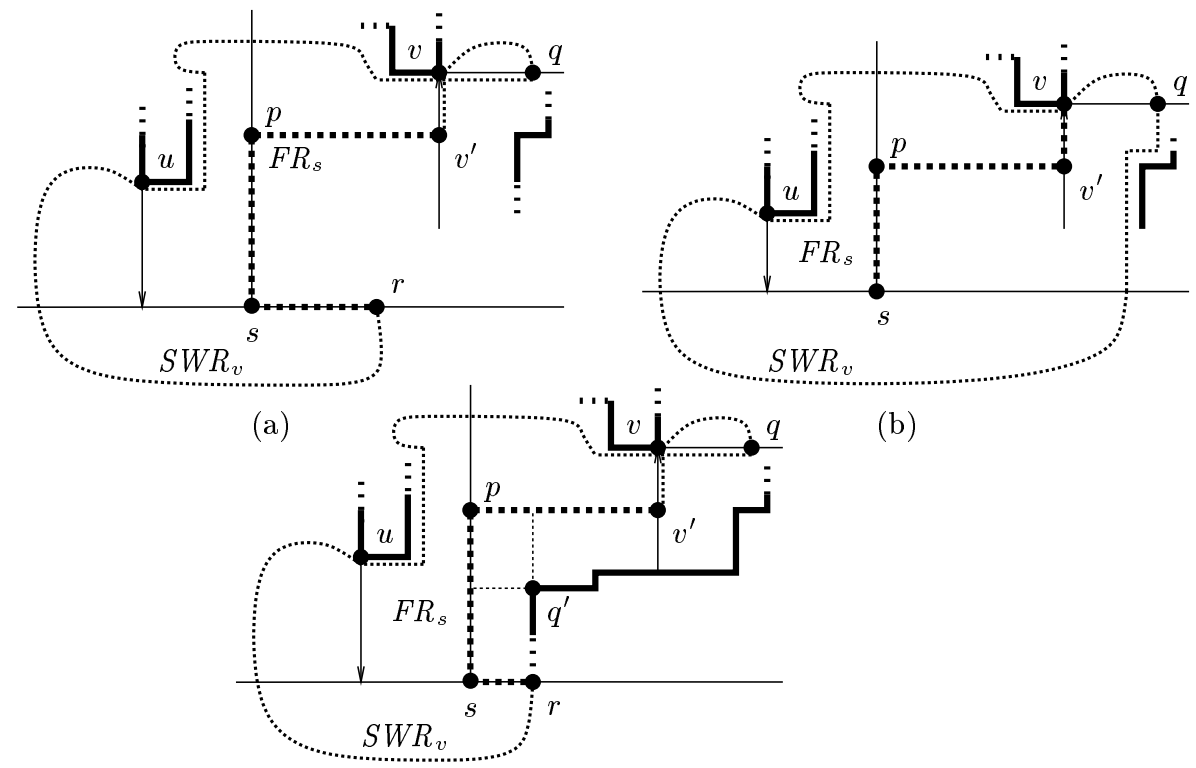

(b)

(c)

Figure 5: Illustrating the proof of Lemma 4.4.

We have that

$$
\min \left\{\|s, q\|_{x},\|s, v\|\right\} \leq\left(\|s, q\|_{x}+\|s, v\|\right) / 2 \leq\left(\|s, q\|_{x}+\|s, v\|_{y}+\|s, u\|_{x}\right) / 2 \leq \text { length }\left(S W R_{s}\right) / 4
$$

proving the bound in this case. The last inequality follows from Lemma 4.2.

If $F R_{s}$ does not pass through $v$; see Figure $6(\mathrm{~d})$; then the tour followed equals $F R_{s}=S P\left(s, v^{\prime}\right) \cup$ $S W R_{v^{\prime}}\left(v^{\prime}, r, a w\right) \cup S P(r, s)$ where $r$ is the last intersection point of $F R_{s}$ with the horizontal line through $s$. The length of $F R_{s}$ is

$$
\operatorname{length}\left(F R_{s}\right)=\operatorname{length}\left(S W R_{v^{\prime}}\right)+2\left\|s, v^{\prime}\right\|_{x} \leq \operatorname{length}\left(S W R_{s}\right)+\left\|s, v^{\prime}\right\|_{x}+\|s, u\|_{x} \leq \frac{3}{2} \operatorname{length}\left(S W R_{s}\right) .
$$

The inequalities follow from Lemmas 4.1 and 4.2 and that $\left\|s, v^{\prime}\right\|_{y} \geq 0$, which concludes the proof.

We have proved the following theorem.

THEOREM 1 CGO is 3/2-competitive.

\section{The Path Problem}

Consider now the situation in which we, instead of a closed tour, wish to obtain a shortest path that explores the interior of our rectilinear polygon, i.e., the path followed by the robot does not have to end at the starting point. Let $O P T_{s}^{P}$ be a shortest exploration path beginning at $s$. It follows immediately that length $\left(O P T_{s}^{P}\right) \geq \operatorname{length}\left(S W R_{s}\right) / 2$, since following the path $O P T_{s}^{P}$ to its end point and then back to $s$ yields a tour having twice the length of the path. 

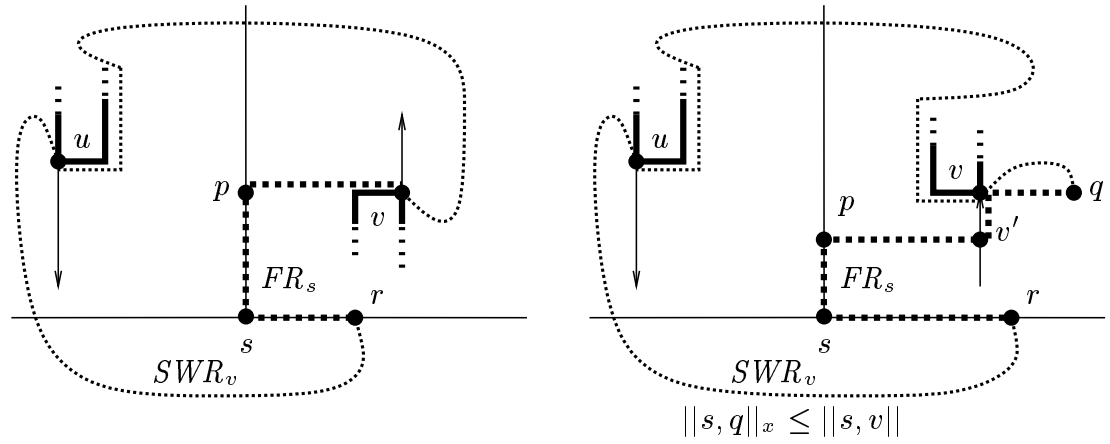

(a)

(b)

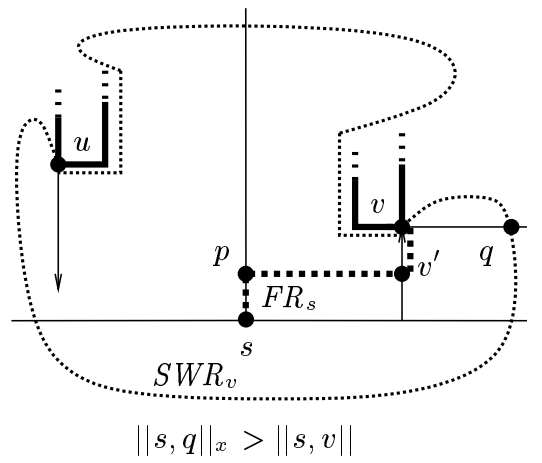

(c)

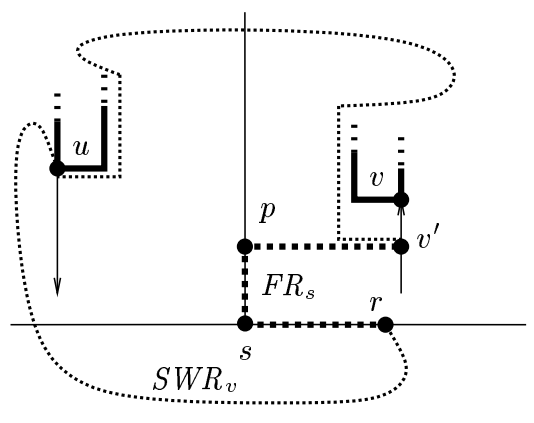

(d)

Figure 6: Illustrating the cases in the proof of Lemma 4.5. 


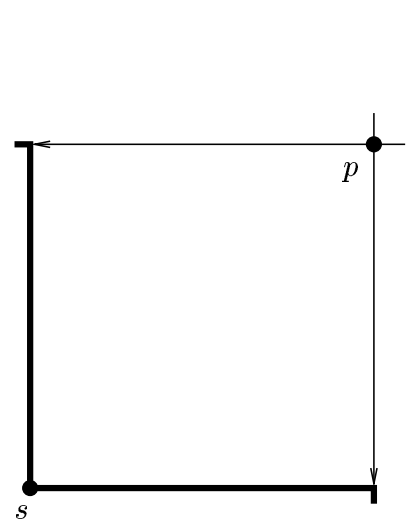

(a)

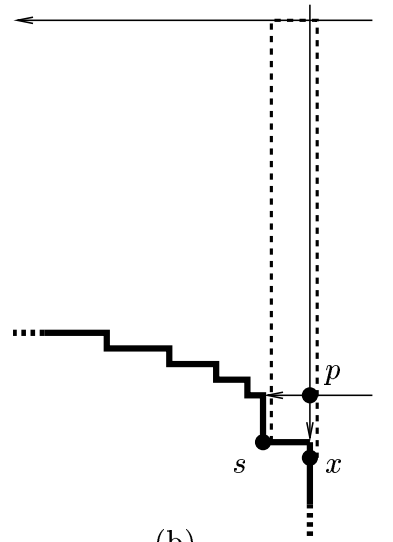

(b)

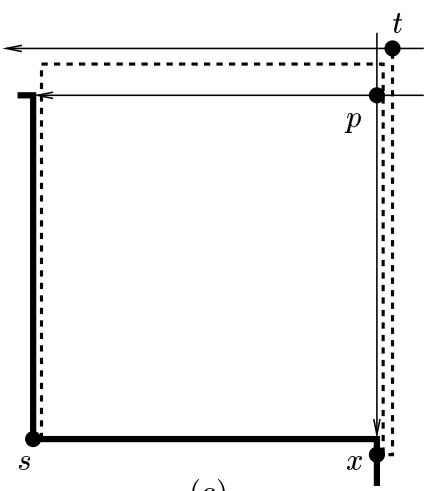

(c)

Figure 7: Illustrating the proof of Theorem 2.

From this we deduce that the strategy CGO presented previously is 3-competitive for path exploration. We continue to show that any strategy for path exploration must be at least 2-competitive.

Theorem 2 There is no deterministic strategy for path exploration of a rectilinear polygon that has competitive ratio $2-\epsilon$ for any $\epsilon>0$.

Proof: We construct a counterexample as in Figure 7. The starting point for the robot is at the lower left corner of the polygon and it essentially sees only the two walls adjacent to it; see Figure 7(a).

The robot now has to move to one of the extensions that it sees. These are both at distance 1 from the starting point. Assume without loss of generality that it moves to the horizontal extension, then the robot realizes that there is a further horizontal extension at distance $\delta$ above it. The robot has the option of either continuing upwards until it has visited all the horizontal extensions (of which the corresponding frontier point is only visible from the previous extension and the extensions are only separated by a distance of $\delta$ ); see Figure 7 (b). This option will make the robot move a distance of $1 / \delta$ upwards until it reaches the last extension and moves to the vertical extension where it realizes that there is a notch at point $x$ (which lies at distance $\delta$ below the starting point $s$ ) forcing it to move back down to this point. The total distance moved is $2 / \delta+1+\delta$.

The optimal path is to move to the vertical extension first, visit the notch at $x$ and then move upwards until all horizontal extensions have been visited, requiring only a distance of $1+2 \delta+1 / \delta$ to be moved. The ratio becomes

$$
\frac{2 / \delta+1+\delta}{1 / \delta+1+2 \delta}=2-\frac{\delta+3 \delta^{2}}{1+\delta+2 \delta^{2}} \geq 2-\epsilon
$$

if $\delta \leq \epsilon / 4<1 / 4$.

On the other hand, if the robot at some point after moving to the first horizontal extension decides to move to the vertical extension it then realizes that it has to move back to the notch at $x$. If it decides to continue upwards until all horizontal extensions have been visited we have the previous situation. If the robot decides at some point to move downwards to visit the notch at $x$, then it has to move back 
up again to visit the next horizontal extension which also turns out to be the last one and the robot terminates at point $t$; see Figure 7(c). Assume that the robot has moved a vertical distance of $D \geq 1$ when it decides to move down and visit the notch at $x$. The total distance moved is then $3 D+1+3 \delta$ whereas the optimal path has length at most $D+1+3 \delta$ and the ratio becomes

$$
\frac{3 D+1+3 \delta}{D+1+3 \delta}=3-\frac{2+6 \delta}{D+1+3 \delta} \geq 2-\epsilon
$$

if $\delta \leq \epsilon / 2<1 / 2$, thus concluding the proof.

\section{Exploration with Multiple Robots}

We now look at the situation when several robots together are required to explore a rectilinear polygon. Again we look at the tour variant, i.e., each robot must terminate the axploration at the starting point. We give upper and lower bounds for the situation with two and three robots all starting at the same point. the measure that we optimize on is the length of the longest tour that any of the robots follow.

Let $O P T_{s}^{k}$ be the tour of the robot that moves the longest length of all the $k$ robots. Since a single robot can follow each of the tours that the $k$ robots follow and thus get a watchman route, we have that length $\left(O P T_{s}^{k}\right) \geq \operatorname{length}\left(S W R_{s}\right) / k$.

We prove lower bounds on the competitive ratio of any exploration strategy using two or three robots.

THEOREM 3 There are no deterministic strategies for exploring a rectilinear polygon with two or three robots having smaller competitive ratio than $3 / 2$.

Proof: We first show the lower bound for two robots and later extend it for three robots. The lower bound is based on essentially the same counterexample that Kleinberg uses for the lower bound for a single robot [7].

The initial polygon is given in Figure 8(a) and consists of a square with notches in the corners. The length of the sides of the square is 2 . Each of the two robots has to move a distance of 2 before it gets to a corner of the polygon and furthermore at most two complete corners can be seen by the robots. Hence placing a notch in one of the corners that is not yet completely seen requires one of the robots to move 6 units whereas the optimal motion can be done with only 4 units, thus proving the result.

The same proof actually goes through for three robots once you realize that independently of how the three robots start their exploration we can force one of them to move 6 units placing at most three notches as shown in Figure 8(b).

We also show a strategy for two robots that has competitive ratio 2. We call the strategy TGO (two-robot GO) since it is based on the GO-strategy of Deng et al; [4]. The two robots each run $\operatorname{IGO}(s$, north, orient $)$, one with orient $=c w$ and the other with orient $=a w$ until the two robots have seen the complete polygon after which they both move back to the starting point.

THEOREM 4 The strategy TGO is 2-competitive for exploration of a rectilinear polygon with two robots. 


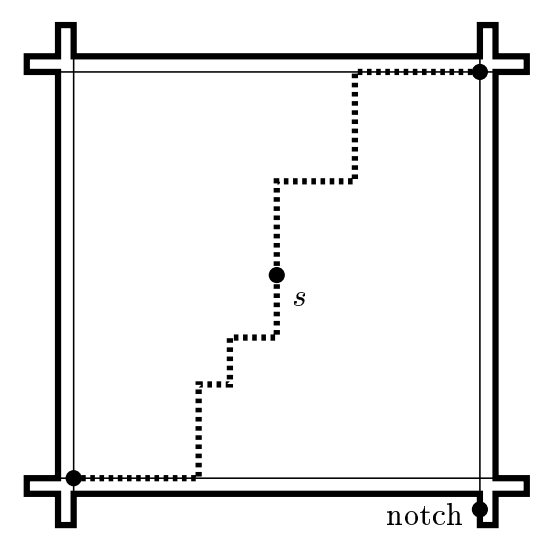

(a)

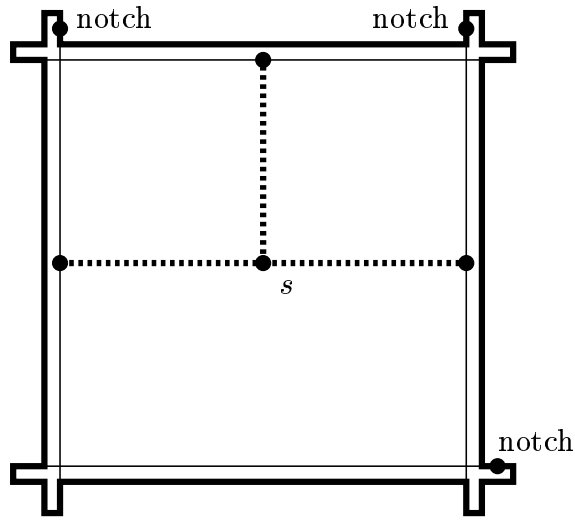

(b)

Figure 8: Illustrating the proof of Theorem 3.

Proof: Let $r$ be the intersection point of $S W R_{s}$ and the vertical axis issuing from $s$ upwards. The intersection point $r$ lies at distance $D \geq 0$ from $s$; see Figure 9 (a). We can view the strategy TGO as first moving the two robots from $s$ to $r$ and then separating, one moving clockwise and the other counterclockwise, following $S W R_{r}$ in two directions until the robots have seen all of the polygon and move back to $s$.

Consider now the robot that moves the farthest. Let $t$ be the point of intersection between the robot's walk and the last extension that it visits before it realizes that the whole polygon is explored. The robot then moves the distance $L=D+\operatorname{length}\left(S W R_{r}(r, t, \operatorname{dir})\right)+\operatorname{length}(S P(t, s))$, where $\operatorname{dir}$ is the direction that the robot moves.

Suppose that we follow the tour $S W R_{s}$ from $s$ in the direction that visits the point $r$ before $t$. We can assume that $t$ is a point on $S W R_{s}$ since it is an intersection point with an extension. Assume without loss of generality that this direction is clockwise. We thus have that

$$
\begin{aligned}
L & =D+\operatorname{length}\left(S W R_{r}(r, t, \operatorname{dir})\right)+\operatorname{length}(S P(t, s)) \\
& \leq \text { length }\left(S W R_{s}(s, r, c w)\right)+\text { length }\left(S W R_{s}(r, t, c w)\right)+\operatorname{length}\left(S W R_{s}(t, s, c w)\right) \\
& =\operatorname{length}\left(S W R_{s}\right) \\
& \leq 2 \operatorname{length}\left(O P T_{s}^{2}\right)
\end{aligned}
$$

which proves the result. That the analysis is tight follows from the example in Figure 9(b).

\section{Conclusions}

We have presented constant competitive strategies and lower bounds to explore a rectilinear simple polygon in the $L_{1}$ metric with one or more robots. Unfortunately none of our results are tight so obvious open problems are to reduce the gaps between the lower bounds and the upper bounds. Especially exploration using $k$ robots, for an arbitrary number $k$, needs to be investigated. 


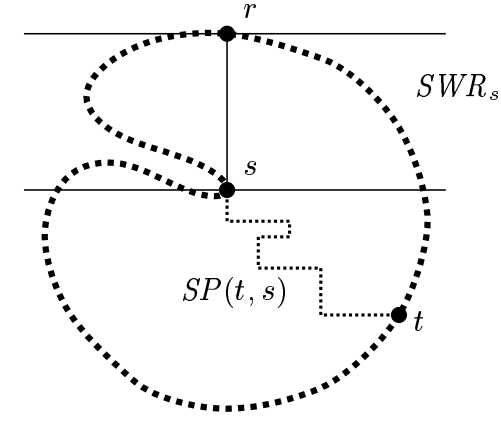

(a)

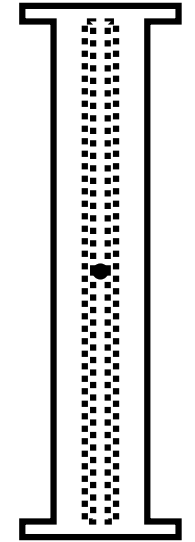

(b)

Figure 9: Illustrating the proof of Theorem 4.

\section{References}

[1] Margrit Betke, Ronald L. Rivest, Mona Singh. Piecemeal Learning of an Unknown Environment. Machine Learning, 18(2-3):231-254, 1995.

[2] K-F. Chan, T. W. LAM. An on-line algorithm for navigating in an unknown environment. International Journal of Computational Geometry \& Applications, 3:227-244, 1993.

[3] W. Chin, S. Ntafos. Optimum Watchman Routes. Information Processing Letters, 28:39-44, 1988.

[4] X. Deng, T. Kameda, C.H. Papadimitriou. How to Learn an Unknown Environment I: The Rectilinear Case. Journal of the ACM, 45(2):215-245, 1998.

[5] M. Hammar, B.J. Nilsson, S. Schuierer. Improved Exploration of Rectilinear Polygons. Nordic Journal of Computing, 9(1):32-53, 2002.

[6] F. Hoffmann, C. Icking, R. Klein, K. Kriegel. The Polygon Exploration Problem. SiaM Journal on Computing, 31(2):577-600, 2001.

[7] J. M. Kleinberg. On-line search in a simple polygon. In Proc. of 5th ACM-SIAM Symp. on Discrete Algorithms, pages 8-15, 1994.

[8] Aohan Mei, Yoshinide Igarashi. An Efficient Strategy for Robot Navigation in Unknown Environment. Inform. Process. Lett., 52:51-56, 1994.

[9] C. H. Papadimitriou, M. Yannakakis. Shortest Paths Without a Map. Theoret. Comput. Sci., 84(1):127-150, 1991. 